\title{
TELEPARALLEL HOMOTHETIC SYMMETRY OF SPECIAL AXIALLY SYMMETRIC STATIC SPACETIME
}

\author{
AMJAD ALI ${ }^{1 *}$, AMJAD MAHMOOD ${ }^{1}$, SUHAIL KHAN ${ }^{2 \#}$ \\ AND MUHAMMAD IQBAL ${ }^{3}$ \\ ${ }^{1}$ Department of Basic Sciences and Islamiat, University of Engineering \& Technology \\ Peshawar, Khyber Pukhtoonkhwa, Pakistan \\ ${ }^{2}$ Department of Mathematics, Abdul Wali Khan University Mardan, Khyber \\ Pukhtoonkhwa Pakistan \\ ${ }^{3}$ Department of Statistics, University of Peshawar, Peshawar Khyber \\ Pukhtoonkhwa, Pakistan. \\ Email:*amjad_puet@yahoo.com, \#suhail_74pk@yahoo.com
}

\begin{abstract}
In this paper we are interested to find teleparallel proper homothetic vector fields over the Lorentzian manifold of special axially symmetric static spacetime. For the purpose we applied teleparallel Lie derivative to the metric for homothetic equations and obtained ten coupled non linear differential equations. These equations are then solved for each possibility of the metric functions and it comes out that only in one case teleparallel proper homothetic vector fields exist for the special choice of metric functions. The spacetime admit eight dimensional teleparallel homothetic vector fields in which one is proper teleparallel homothetic vector and remaining seven are teleparallel Killing vector fields.
\end{abstract}

Keywords: Teleparallel theory, Torsion, Proper teleparallel homothetic vectors.

PACS: 04.20.Jb, 04.20.Cv, 02.30.Jr.

1. Introduction: After the birth of general relativity, our understanding of universe got momentum and different aspects of the universe have been studied through the knowledge provided by this theory. The study of conservation laws for the metric of a spacetime is one aspect that helps us to understand and expose the hidden realities of the universe. Since symmetries of a spacetime metric give rise to conservation laws [1], Killing, homothetic, conformal and self-similar symmetries of a spacetime metric are widely studied in the presence of curvature [2-11]. In the past it has been observed that the laws of gravitation can be equivalently described for the spacetime having torsion only [12]. The idea of Killing symmetry in teleparallel theory was introduced by M. Sharif et al [13]. They obtained Killing vectors for Einstein universe in the presence of torsion only. Much work has been done on finding Killing vector fields of different spacetimes in context of teleparallel theory of gravitation [14-18]. Homothetic vector fields are considered as an important symmetry in general relativity, as it preserve the metric up to a constant factor and give an extra symmetry ignored by the Killing symmetry. Homothetic vector fields in the presence of torsion in teleparallel theory are obtained in [19-22]. In order to understand the physical and geometrical aspects of our mysterious universe much work is needed to be done through different symmetries in the presence of curvature or torsion only. In this paper homothetic vector fields in context of teleparallel theory has been investigated for special axially symmetric static spacetime. The desired results are obtained for the special choice of the metric functions involved. In the following we are giving a brief introduction of teleparallel theory and then in next section teleparallel homothetic vector fields are obtained. 


\section{OVERVIEW:}

Covariant derivative $\nabla_{\rho}$ in teleparallel theory for a covariant tensor of rank 2 is defined as [23]

$$
\nabla_{\rho} B_{\mu v}=B_{\mu v, \rho}-\Gamma_{\rho v}^{\theta} B_{\mu \theta}-\Gamma_{\mu \rho}^{\theta} B_{v \theta},
$$

where comma is used for partial derivative and $\Gamma^{\theta}{ }_{\rho v}$ is a Weitzenböck connection and defined as [23]

$$
\Gamma_{\mu v}^{\theta}=S_{a}^{\theta} \partial_{v} S_{\mu}^{a},
$$

here the non trivial tetrad field $S^{a}{ }_{\mu}$ and its inverse field $S_{a}{ }^{v}$ satisfy the relations

$$
S^{a}{ }_{\mu} S_{a}{ }^{v}=\delta_{\mu}{ }^{v}, \quad S^{a}{ }_{\mu} S_{b}{ }^{\mu}=\delta_{b}{ }^{a}
$$

The tetrad fields defined above generate Riemannian metric as

$$
g_{\mu v}=\eta_{a b} S^{a}{ }_{\mu} S^{b}{ }_{\nu},
$$

where $\eta_{a b}$ is the Minkowski metric $\eta_{a b}=\operatorname{diag}(-1,1,1,1)$. An anti symmetric tensor in the lower indices known as torsion tensor is defined as [23]

$$
T^{\theta}{ }_{\mu \nu}=\Gamma^{\theta}{ }_{\nu \mu}-\Gamma_{\mu \nu}^{\theta},
$$

A vector field $X$ is said to be teleparallel homothetic vector field if it satisfies equation

$$
L_{X}^{T} g_{\mu v}=g_{\mu v, \rho} X^{\rho}+g_{\rho v} X_{, \mu}^{\rho}+g_{\mu \rho} X_{, v}^{\rho}+X^{\rho}\left(g_{\theta v} T^{\theta}{ }_{\mu \rho}+g_{\mu \theta} T^{\theta}{ }_{\nu \rho}\right)=2 \alpha g_{\mu v} \text {, }
$$

where $L_{X}^{T}$ represents Lie derivative in teleparallel theory along the vector field $X$ and $\alpha \in R \backslash\{0\}[21]$.

\section{MAIN RESULTS:}

Consider special axially symmetric static spacetime in usual coordinates $(t, r, \theta, \phi)$ (labeled by $\left(x^{0}, x^{1}, x^{2}, x^{3}\right)$, respectively) with the line element [24]

$$
d s^{2}=-e^{A(r, \theta)} d t^{2}+e^{B(r, \theta)}\left(d r^{2}+d \theta^{2}+d \varphi^{2}\right),
$$

where $A$ and $B$ are functions of $r$ and $\theta$ only. Spacetime (7) admit two linearly independent Killing vector fields in general relativity, which are $\frac{\partial}{\partial t}$ and $\frac{\partial}{\partial \phi}$. Using relation (4) the components of tetrad and its inverse can be obtained as follows

$$
S_{u}^{a}=\left[\begin{array}{cccc}
e^{\frac{1}{2} A(r, \theta)} & o & o & o \\
o & e^{\frac{1}{2} B(r, \theta)} & o & o \\
o & o & e^{\frac{1}{2} B(r, \theta)} & o \\
o & o & o & e^{\frac{1}{2} B(r, \theta)}
\end{array}\right]
$$




$$
S_{a}^{u}=\left[\begin{array}{cccc}
e^{-\frac{1}{2} A(r, \theta)} & o & o & o \\
o & e^{-\frac{1}{2} B(r, \theta)} & o & o \\
o & o & e^{-\frac{1}{2} B(r, \theta)} & o \\
o & o & o & e^{-\frac{1}{2} B(r, \theta)}
\end{array}\right]
$$

It can be verified easily that equations (3) and (4) between $S^{a}{ }_{\mu}$ and $S_{a}{ }^{\mu}$ are satisfied. Using equation (2), the corresponding non-vanishing Weitzenböck connections are obtained as

$$
\begin{aligned}
& \Gamma_{01}^{0}=\frac{1}{2} \mathrm{~A}_{\mathrm{r}}(\mathrm{r}, \theta), \quad \Gamma_{02}^{0}=\frac{1}{2} \mathrm{~A}_{\theta}(\mathrm{r}, \theta), \Gamma_{12}^{1}=\frac{1}{2} \mathrm{~B}_{\theta}(\mathrm{r}, \theta), \\
& \Gamma_{21}^{2}=\frac{1}{2} \mathrm{~B}_{\mathrm{r}}(\mathrm{r}, \theta), \quad \Gamma_{31}^{3}=\frac{1}{2} \mathrm{~B}_{\mathrm{r}}(\mathrm{r}, \theta), \quad \Gamma_{32}^{3}=\frac{1}{2} \mathrm{~B}_{\theta}(\mathrm{r}, \theta)
\end{aligned}
$$

Thus the non vanishing torsion components by using equation (5) are

$$
\begin{aligned}
& \mathrm{T}_{01}^{0}=\frac{-1}{2} \mathrm{~A}_{\mathrm{r}}(\mathrm{r}, \theta)=-\mathrm{T}_{10}^{0}, \mathrm{~T}_{02}^{0}=\frac{-1}{2} \mathrm{~A}_{\theta}(\mathrm{r}, \theta)=-\mathrm{T}_{20}^{0}, \\
& \mathrm{~T}_{12}^{1}=\frac{-1}{2} \mathrm{~B}_{\theta}(\mathrm{r}, \theta)=-\mathrm{T}_{21}^{1} \mathrm{~T}_{12}^{2}=\frac{1}{2} \mathrm{~B}_{\mathrm{r}}(\mathrm{r}, \theta)=-\mathrm{T}_{21}^{2}, \\
& \mathrm{~T}_{13}^{3}=\frac{1}{2} \mathrm{~B}_{\mathrm{r}}(\mathrm{r}, \theta)=-\mathrm{T}_{31}^{3}, \mathrm{~T}_{23}^{3}=\frac{1}{2} \mathrm{~B}_{\theta}(\mathrm{r}, \theta)=-\mathrm{T}_{32}^{3} .
\end{aligned}
$$

A vector field $X$ is said to be teleparallel homothetic vector field if it satisfies equation (6). One can write (6) explicitly using (7) and (11) as:

$$
\begin{gathered}
X_{, 0}^{0}=\alpha, \\
X_{, 1}^{0}+\frac{1}{2} \mathrm{~A}_{\mathrm{r}}(\mathrm{r}, \theta) \mathrm{X}^{0}-\mathrm{e}^{\mathrm{B}(\mathrm{r}, \theta)-\mathrm{A}(\mathrm{r}, \theta)} X_{, 0}^{1}=0, \\
X_{, 2}^{0}+\frac{1}{2} \mathrm{~A}_{\theta}(\mathrm{r}, \theta) \mathrm{X}^{0}-\mathrm{e}^{(\mathrm{B}(\mathrm{r}, \theta)-\mathrm{A}(\mathrm{r}, \theta))} X_{, 0}^{2}=0, \\
X_{, 3}^{0}-\mathrm{e}^{(\mathrm{B}(\mathrm{r}, \theta)-\mathrm{A}(\mathrm{r}, \theta))} X_{, 0}^{3}=0, \\
X_{, 1}^{1}+\frac{1}{2} \mathrm{~B}_{\mathrm{r}}(\mathrm{r}, \theta) \mathrm{X}^{1}=\alpha, \\
X_{, 2}^{1}+\frac{1}{2} \mathrm{~B}_{\theta}(\mathrm{r}, \theta) \mathrm{X}^{1}+X_{, 1}^{2}+\frac{1}{2} \mathrm{~B}_{\mathrm{r}}(\mathrm{r}, \theta) \mathrm{X}^{2}=0,
\end{gathered}
$$




$$
\begin{aligned}
& X_{, 3}^{1}+X_{, 1}^{3}+\frac{1}{2} \mathrm{~B}_{\mathrm{r}}(\mathrm{r}, \theta) X^{3}=0 \\
& X_{, 2}^{2}+\frac{1}{2} \mathrm{~B}_{\theta}(\mathrm{r}, \theta) X^{2}=\alpha \\
& X_{, 3}^{2}+X_{, 2}^{3}+\frac{1}{2} \mathrm{~B}_{\theta}(\mathrm{r}, \theta) X^{3}=0, \\
& X_{, 3}^{3}=\alpha .
\end{aligned}
$$

In order to get teleparallel homothetic vector fields from the above equations we proceed as follows:

Solving equations (12), (13), (20) and (21) respectively, a system of equations is formed

$$
\begin{aligned}
\mathrm{X}^{0}= & \alpha \mathrm{t}+\mathrm{E}^{1}(\mathrm{r}, \theta, \varphi), \\
\mathrm{X}^{1}= & \mathrm{te}^{(\mathrm{A}(\mathrm{r}, \theta)-\mathrm{B}(\mathrm{r}, \theta))} E_{\mathrm{r}}^{1}(\mathrm{r}, \theta, \varphi)+\frac{\alpha \mathrm{t}^{2}}{4} \mathrm{e}^{(\mathrm{A}(\mathrm{r}, \theta)-\mathrm{B}(\mathrm{r}, \theta))} \mathrm{A}_{\mathrm{r}}(\mathrm{r}, \theta) \\
& +\frac{\mathrm{t}}{2} \mathrm{e}^{(\mathrm{A}(\mathrm{r}, \theta)-\mathrm{B}(\mathrm{r}, \theta))} \mathrm{A}_{\mathrm{r}}(\mathrm{r}, \theta) \mathrm{E}^{1}(\mathrm{r}, \theta, \varphi)+\mathrm{E}^{3}(\mathrm{r}, \theta, \varphi), \\
\mathrm{X}^{2}= & -\varphi E^{2} \theta(\mathrm{t}, \mathrm{r}, \theta)-\frac{\alpha \varphi^{2}}{4} \mathrm{~B}_{\theta}(\mathrm{r}, \theta)-\frac{\varphi}{2} \mathrm{~B}_{\theta}(\mathrm{r}, \theta) \mathrm{E}^{2}(\mathrm{t}, \mathrm{r}, \theta)+\mathrm{E}^{4}(\mathrm{t}, \mathrm{r}, \theta), \\
\mathrm{X}^{3}= & \alpha \varphi+\mathrm{E}^{2}(\mathrm{t}, \mathrm{r}, \theta),
\end{aligned}
$$

where $\mathrm{E}^{1}(\mathrm{r}, \theta, \varphi), \mathrm{E}^{2}(\mathrm{t}, \mathrm{r}, \theta), \mathrm{E}^{3}(\mathrm{r}, \theta, \varphi)$ and $\mathrm{E}^{4}(\mathrm{t}, \mathrm{r}, \theta)$ are functions of integration. These unknown functions will be determined by the use of remaining six equations. Now using the system of equations (22) in equations (15), (16) and (18) respectively and solving, we reach to five different cases. We will discuss each case in turn.

\section{Case I:}

In this case the metric functions of (7) takes the form $A(r, \theta)=\operatorname{In}\left(r / 2-c_{4} \theta-c_{5}\right)^{2}+c_{3}$ and

$\mathrm{B}(\mathrm{r}, \theta)=\operatorname{In}\left(r / 2-\mathrm{c}_{4} \theta-\mathrm{c}_{5}\right)^{2}+\mathrm{c}_{2}$, where $\mathrm{c}_{2}, c_{3}, \mathrm{c}_{4}, \mathrm{c}_{5} \in \mathrm{R}\left(\mathrm{c}_{4} \neq 0, \mathrm{c}_{2} \neq \mathrm{c}_{3}\right)$. Using these information and system of equations (22) in the remaining three equations (14), (17) and (19) respectively. After a lengthy calculation, solution of equations (12)-(21) is obtained as

$$
\begin{aligned}
& X^{\mathrm{O}}=\alpha \boldsymbol{t}+e^{\frac{-\mathbf{1}}{2} \boldsymbol{A}(\boldsymbol{r}, \boldsymbol{\theta})} \boldsymbol{c}_{19} \\
& X^{1}=e^{\frac{-1}{2} B(r, \theta)}\left[\alpha\left(\frac{t^{2} c_{20}}{4}-\frac{\phi^{2} c_{21}}{4}-\frac{\theta^{2} c_{21}}{4}-r \theta c_{22}+\frac{r^{2} c_{21}}{4}-r c_{23}\right)-\frac{\phi c_{25}}{2}-\theta c_{26}+c_{27}\right] \\
& X^{2}=e^{\frac{-1}{2} B(r, \theta)}\left[\alpha\left(\frac{\phi^{2} c_{22}}{2}-\frac{t^{2} c_{28}}{2}+\frac{r^{2} c_{22}}{2}+\frac{r \theta c_{21}}{2}-\frac{\theta^{2} c_{22}}{2}-\theta c_{23}\right)+\phi c_{29}+r c_{26}+c_{31}\right] \\
& X^{3}=\alpha \phi+e^{\frac{-1}{2} B(r, \theta)}\left[-\theta c_{29}+\frac{1}{2} r c_{25}+c_{33}\right]
\end{aligned}
$$

In order to find proper teleparallel homothetic vector field we subtract Killing vector fields form (23) to get

$$
\begin{aligned}
& X^{\mathbf{O}}=\boldsymbol{\alpha t} \\
& X^{1}=e^{\frac{-1}{2} B(r, \theta)}\left[\alpha\left(\frac{t^{2} c_{20}}{4}-\frac{\phi^{2} c_{21}}{4}-\frac{\theta^{2} c_{21}}{4}-r \theta c_{22}+\frac{r^{2} c_{21}}{4}-r c_{23}\right)\right]
\end{aligned}
$$




$$
\begin{aligned}
& X^{2}=e^{\frac{-1}{2} B(r, \theta)}\left[\alpha\left(\frac{\phi^{2} c_{22}}{2}-\frac{t^{2} c_{28}}{2}+\frac{r^{2} c_{22}}{2}+\frac{r \theta c_{21}}{2}-\frac{\theta^{2} c_{22}}{2}-\theta c_{23}\right)\right] \\
& X^{3}=\alpha \phi
\end{aligned}
$$

Case II:

In this case the metric functions of (7) takes the form $A(r, \theta)=-2 \log \left(\frac{r}{2}-I^{2}(\theta)\right)+I^{16}(\theta)$ and $\mathrm{B}(\mathrm{r}, \theta)=\operatorname{In}\left(r / 2-\mathrm{c}_{4} \theta-\mathrm{c}_{5}\right)^{2}+\mathrm{c}_{2}$, where $\mathrm{c}_{4}, \mathrm{c}_{5} \in \mathrm{R}\left(\mathrm{c}_{4} \neq 0\right)$. Using these information and system of equations (22) in the remaining three equations (14), (17) and (19) respectively. After a lengthy calculation, we reach to an equation of the form $\frac{-2 \mathrm{e}^{\left(\mathbf{I}^{16}(\theta)-\mathrm{c}_{2}\right)}}{\left(\frac{\mathbf{r}}{2}-\mathbf{I}^{2}(\theta)\right)^{6}}=0$, which clearly gives contradiction and so this case is not possible.

\section{Case III:}

In this case $A=A(\theta), B=B(r, \theta)$, when we put back these information along with system of equations (22) we get an equation of the form

$$
\frac{e^{\left(\frac{1}{4} D_{3}-\frac{3}{4} D_{2}+D_{1}\right)}}{\left(\frac{r}{2}-D_{4}\right)^{\frac{7}{2}}}=0
$$

which again gives contradiction and hence this case is also not possible.

\section{Case IV:}

In this case $A=A(r, \theta), B=B(\theta)$. When we put back these information along with system of equations (22) we reach to an equation of the form

$$
\frac{e^{L_{(\theta)}^{8}}}{2\left(r-L^{6}(\theta)\right)^{2}}=0
$$

which gives contradiction and hence this case is also not possible.

It is also important to note that in the case $A=A(\theta), B=B(\theta)$ we once again reach to an equation which leads us to contradiction. Hence the spacetime admit teleparallel homothetic vector field in one case for the special choice of metric functions.

\section{REFERENCES}

[1] Petrov, A. Z. (1969). Physics Einstein spaces. Pergamon, Oxford University Press.

[2] Ali, A., Hussain, T. and Khan, J. (2012). Isometries in Bianchi types $V I$ 。 and VII。 space-times. Advanced Studies in Theoretical Physics. 6, 607-613.

[3] Ali, A., Kamran and Minullah, Z. (2012). Classification of spherically symmetric non static spacetimes according to their Killing vector fields. Applied Mathematical Sciences. 6, 2681-2686.

[4] Ali, A., Khan, J. and Hussain, T. (2012). Proper homothetic symmetry in Bianchi type IV space-times. Advanced Studies in Theoretical Physics. 6, 187-192.

[5] Ali, A., Minullah Z. and Kamran. (2012). Proper homothetic vector field in Bianchi type-V space-times, Advanced Studies in Theoretical Physics. 6, 193-200.

[6] Shabbir, G., Ramzan, M. and Ali, A. (2009). Classification of non static spherically symmetric spacetimes according to their proper conformal vector fields. University Polithnica of Bucharest scientific bulletin series A-Applied Mathematics and Physics. 71(1), 3-8.

[7] Shabbir, G. and Ali, A. (2009). A note on proper conformal symmetry in Bianchi types VIII and IX space-times. Advanced Studies in Theoretical Physics. 3, 93-97. 
[8] Shabbir, G. and Ali, A. (2009). Classification of spatially homogeneous rotating space-times according to their conformal vector fields. Applied Mathematical Sciences. 3(18), 869-876.

[9] Shabbir, G. and Khan, S. (2010). A note on self similar vector fields in plane symmetric static spacetimes. TWMS Journal of Pure and Applied Mathathematics. 1, 252-256.

[10] Shabbir, G. and Khan, S. (2012). A note on self-similar vector fields in static spherically symmetric spce-times. University Polithnica of Bucharest scientific bulletin series A-Applied Mathematics and Physics. 74(4), 177-182.

[11] Shabbir, G. and Khan, S. (2013). A note on self-similar vector fields in cylindrically symmetric static space-times. TWMS Journal of Pure and Applied Mathathematics. 04, 38-43.

[12] Aldrovandi, R. and Pereira, J. G. (1995). An introduction to geometrical physics, World Scientific.

[13] Sharif, M. and Amir, M. J. (2008). Teleparallel Killing vectors of the Einstein universe. Modern Physics Letters A. 23(13), 963-969.

[14] Shabbir, G., Khan, S. and Ali, A. (2011). A note on classification of spatially homogeneous rotating space-times according to their teleparallel Killing vector fields in teleparallel theory of gravitation. Communications in Theoretical Physics, 55(2), 268-272.

[15] Shabbir, G., Ali, A. and Khan, S. (2011). A note on teleparallel Killing vector fields in Bianchi type VIII and IX space-times in teleparallel theory of gravitation. Chinese Physics B. 20, 070401-070406.

[16] Shabbir, G. and Khan, S. (2010). A note on Killing vector fields of Bianchi type II space-times in teleparallel theory of gravitation. Modern Physics Letters A. 25, 1733-1740.

[17] Shabbir, G. and Khan, S. (2010). Classification of cylindrically symmetric static space-times according to their Killing vector fields in teleparallel theory of gravitation. Modern Physics Letters A. 25, 525-533.

[18] Shabbir, G., Khan, S. and Amir, M. J. (2011). A note on classification of cylindrically symmetric non static space-times according to their teleparallel Killing vector fields in the teleparallel theory of gravitation. Brazilian Journal of Physics. 41, 184-194.

[19] Shabbir, G. and Khan, S. (2010). Classification of Bianchi type I space-times according to their proper teleparallel homothetic vector fields in the teleparallel theory of gravitation. Modern Physics Letters A. 25, 2145-2153.

[20] Shabbir, G. and Khan, S. (2010). Classification of teleparallel homothetic vector fields in cylindrically symmetric static space-times in teleparallel theory of gravitation. Communications in Theoretical Physics. $54,675-678$.

[21] Shabbir, G. and Khan, S. (2012). A note on proper teleparallel homothetic vector fields in non static plane symmetric Lorentzian manifold. Romanian Journal of Physics. 57, 571-581.

[22] Khan, S., Hussain, T. and Khan, G. A. (2013). A note on proper teleparallel homothetic motions of well-known spacetime using non diagonal tetrad. Life Science Journal. 10 (11s), 87-90.

[23] Aldrovendi, R. and Pereira, J. G. (2001). An introduction to gravitation theory. Preprint.

[24] Stephani, H., Kramer, D., Maccallum, M., Hoenselaers C. and Herlt, E. (2003). Exact Solutions of Einstein's Field Equations (Second Edition), Cambridge University Press. 